\title{
La educación inclusiva en instituciones formadoras de maestros. Balance de la experiencia de la Universidad Pedagógica Nacional de Colombia ${ }^{1}$
}

\author{
Inclusive Education in Teacher Training Institutions. \\ Balance of National Pedagogical University of Colombia's Experience
}

Olga Cecilia Díaz Flórez, Luz Mary Lache Rodríguez, Isabel Garzón Barragán y Adolfo León Atehortúa Cruz²

\section{Resumen}

Este artículo presenta un balance de los desarrollos de la Universidad Pedagógica Nacional de Colombia (UPN) en el último quinquenio en relación con políticas institucionales, cambios normativos, estrategias y acciones generadas para la formación de maestros, en la perspectiva de la educación superior inclusiva (ESI). A partir de este balance, se desarrolla el contraste con las formulaciones que al respecto hacen las políticas nacionales e internacionales en dos ámbitos de análisis: la educación inclusiva y la formación de maestros en esta última década, que permiten identificar las tensiones en las que se desarrollan las políticas y su interacción con las exigencias estatales, así como los logros que pese a estas tensiones ha producido la Universidad. Las principales tensiones se encuentran entre el desarrollo de una política integral y flexible de educación inclusiva y las condiciones que ofrece el Estado a las Instituciones de Educación Superior (IES) públicas en Colombia; la ausencia de coherencia entre las políticas de formación de maestros y las de educación inclusiva en la educación superior y en cuanto al papel del Estado en términos de su corresponsabilidad en el desarrollo de las políticas públicas.

\section{Palabras clave}

Formación de maestros, educación inclusiva, políticas educativas, educación superior, calidad educativa.

\section{Abstract}

This article presents a balance of the advances of the National Pedagogical University (UPN) of Colombia in the last five years in relation to institutional policies, normative changes, strategies and actions generated for teacher training, in the perspective of inclusive higher education. This balance is contrasted with the formulations made by national and international policies in two areas of analysis: inclusive education and teacher training in the last decade, which allow identifying the tensions in which the policies are implemented and their interaction with the state requirements, as well as the achievements that, despite of these tensions, the UPN has produced. The main tensions are between the development of an integral and flexible inclusive education policy and the conditions offered by the State to public higher education institutions in Colombia; the lack of coherence between the teacher training policies and those of inclusive education in higher education and the role of the State in terms of its co-responsibility in the implementation of these public policies.

\section{Keywords}

Teacher training, inclusive education, educational policies, higher education, educational quality.

\section{Cómo citar/Citation}

Díaz Flórez, Olga Cecilia; Lache Rodríguez, Luz Mary; Garzón Barragán, Isabel y Atehortúa Cruz, Adolfo León (2019). La educación inclusiva en instituciones formadoras de maestros. Balance de la experiencia de la Universidad Pedagógica Nacional de Colombia. Revista de Sociología de la Educación-RASE, 12 (2), 318-336. http://dx.doi.org/10.7203/RASE.12.2.14814.

1 Este artículo forma parte del proyecto TO-INN From Tradition to Innovation in Teacher Training Institutions financiado por la Unión Europea a través del programa Erasmus, Acción K2 Capacity Building, (573685-EPP-2016-1-ES-EPPKA2-CBHE-JP) https://www.toinn.org.

2 Olga Cecilia Díaz Flórez, odiaz@pedagogica.edu.co; Luz Mary Lache Rodríguez, lmlacher@pedagogica.edu.co; Isabel Garzón Barragán, igarzon@pedagogica.edu. co; Adolfo León Atehortúa Cruz, adolate@pedagogica.edu.co. Todos de la Universidad Pedagógica Nacional de Colombia. 
Olga Cecilia Díaz Flórez, Luz Mary Lache Rodríguez, et al. La educación inclusiva en instituciones formadoras de maestros. Balance de la experiencia de la Universidad Pedagógica Nacional de Colombia

\section{Introducción}

El presente artículo se orienta a elaborar un análisis de las políticas institucionales de educación inclusiva y de las acciones generadas por una institución formadora de maestros, la Universidad Pedagógica Nacional de Colombia (UPN), a partir del balance de los desarrollos del último quinquenio en términos de sus alcances y limitaciones. Los referentes para elaborar este balance serán, tanto la articulación entre planteamientos de la política institucional y sus concreciones en las acciones realizadas, como el contraste con las formulaciones que al respecto hacen las políticas nacionales e internacionales en los dos ámbitos de análisis: la educación inclusiva y la formación de maestros, en esta última década. A partir de estos contrastes, se identifican tensiones en las que se desarrollan las políticas y su interacción con las exigencias estatales.

En relación con el balance, en primera instancia, el artículo contextualiza las políticas institucionales, su horizonte y compromiso con lo que implica configurar un proyecto de formación de maestros -Proyecto Educativo Institucional (PEI)-, así como sus desarrollos más recientes con respecto al Plan de Desarrollo Institucional (PDI 2014-2019), en torno a las denominadas políticas de educación inclusiva. En la segunda parte se presenta el debate acerca de las tensiones que se configuran al contraponerse las orientaciones y pretensiones formuladas en diferentes políticas estatales que dejan a las instituciones de educación superior en disyuntivas de difícil resolución.

\section{Políticas institucionales para favorecer la educación inclusiva}

En los postulados del PEI, desde el año 2010, y en relación con la naturaleza misma de la Universidad, se formuló como una de sus preocupaciones centrales el desarrollo de acciones orientadas a garantizar la inclusión y atender la diversidad, «mediante la dinamización de procesos educativos interculturales, innovadores e investigativos dirigidos en particular a: poblaciones que requieren una atención educativa especial, discapacitados cognitivos, funcionales y talentosos, comunidades indígenas, comunidades afrocolombianas y comunidades campesinas) (UPN, 2010: 11). Bajo esta perspectiva, la UPN se definió como una universidad incluyente, esto es, con «la comprensión de que la especie bumana en su conjunto nos ofrece múltiples formas y condiciones de existir y habitar como sujetos, que están mediadas por razones de origen, etnia, cultura, género, situación social, vulnerabilidad, estado físico, edad, condición psicológica, entre otras) (UPN, 2010: 11).

La inclusión quedó igualmente planteada en este PEI en el marco de los principios y ejes transversales que estructuran los lineamientos para el desarrollo académico institucional y que organizan las dinámicas, programas y proyectos de la comunidad pedagógica. En este sentido, la perspectiva de la inclusión se hizo explícita en torno a los compromisos misionales de docencia, investigación y proyección social que configuraban la apuesta por el fortalecimiento académico de una universidad comprometida «con la formación de maestros, de personas vinculadas con la orientación y desarrollo de la educación y del ser humano en generali (UPN, 2010: 16).

Esta orientación hacia una educación inclusiva permaneció en el PDI 2014-2019, con la aclaración de que no solo se aspiraba a dar continuidad a aquellos programas que se consideran asistencialistas y que ofrecen apoyo directo a la población estudiantil más vulnerable, favoreciendo iniciativas, proyectos y acciones específicas, sino que se esperaba impulsar iniciativas que configuraban una inclusión diferencial en los programas, en el marco de una propuesta de «bienestar universitario para todas y todos».

Desde esta perspectiva, en las formulaciones de este PDI se expresó la necesidad de propiciar una visión de la educación inclusiva más amplia; lo cual implicaba no solo favorecer la adquisición de las mismas 
competencias y conocimientos básicos para el mejor aprovechamiento de nuevas oportunidades, sino también reconocer otros aspectos que involucraran al Estado, al conjunto de la sociedad y a las instituciones educativas: el acceso y la permanencia, impulso a las transformaciones curriculares, flexibilidad en los procesos de aprendizaje, recursos de apoyo, «estimulo y nivelación según los requerimientos de diversas trayectorias formativas de los estudiantes, condiciones de vida universitaria equiparables -en una lógica de nivelación por lo alto-, y relaciones de cooperación entre los actores e instituciones de la educación superion» (UPN, 2014: 39-40).

El PDI subrayó, además, la oportunidad que representaba para la UPN hacerse partícipe de la concreción de los acuerdos de paz que buscaba el país, lo cual ofrecía la posibilidad de generar un «liderazgo en materia de propuestas educativas para la construcción de una paz duradera con justicia social, que posibilitara la incorporación respetuosa de los insurgentes a la vida ciudadana» (UPN, 2014: 43).

En consonancia con estas aspiraciones, se planteó que los principios formulados en el PDI se derivaban de la identidad institucional y de las perspectivas ético-pedagógicas en las que se posicionan sus actores para su elaboración, las cuales se entienden como fundamento para la definición de las acciones institucionales. La manera de enunciar los principios revela, entonces, los alcances de esta apuesta de desarrollo integral de la universidad: Participación y democracia, Convivencia y corresponsabilidad, Interculturalidad y diálogo de saberes, Autonomía y libertad y, Buen vivir y sentido de comunidad.

En este contexto, entre las metas del PDI se plantearon iniciativas como: «Crear tres estrategias de acompañamiento para mejorar la inclusión de estudiantes en condiciones de vulnerabilidad», «mejorar la selección e ingreso de estudiantes a la Universidad», realizar estudios orientados a analizar y mejorar «los índices de deserción, permanencia y graduación de los estudiantes de la Universidad». En estos planteamientos ya podemos identificar un primer nivel sobre el cual formular un balance, pues es evidente que las especificaciones de la política institucional con respecto a las metas, concretan el tímido alcance de las formulaciones antes esbozadas.

En esta dirección, podemos identificar un primer nivel de tensión interna relacionado con los distanciamientos que se generan entre unas aspiraciones generales que se ratifican en los documentos orientadores del desarrollo institucional, y su operativización y concreción en torno a metas, cuyo alcance dista de responder a tales aspiraciones. Una explicación de este distanciamiento se relaciona con los modos en que se elaboran dichos documentos, en tanto sus autores no se responsabilizan de las construcciones y acciones a que invitan con sus textos; más aún, con el carácter participativo generado en su elaboración. Otra explicación se relaciona con la forma medida y sopesada con la cual se proyecta el uso de los recursos financieros y el desarrollo de las políticas institucionales, que se traduce en los alcances de las metas, en especial en una coyuntura como la atravesada en ese momento por la UPN, en la que primaba la incertidumbre respecto a los recursos que el gobierno proyectaba girar a las instituciones de educación superior del sector estatal.

Ahora bien, al indagar por los desarrollos que en los últimos años se han producido en torno a lo que representa una educación inclusiva de carácter integral y articulado, podemos identificar un conjunto de transformaciones y desarrollos que bien vale la pena analizar en el marco de la pretensión que subyace al presente artículo: el reconocimiento de las tensiones y exigencias que representa el desarrollo de una educación inclusiva $-\mathrm{y}$ no solamente una inclusión educativa ${ }^{3}$-, a la vez que destacar las inconsistencias que

3 La diferencia entre estas dos orientaciones radica en que la inclusión educativa se restringe al acceso de grupos poblacionales que están por fuera del marco de la educación para todos y a la adaptación de los estudiantes a las condiciones del sistema educativo, mientras que la educación inclusiva trabaja en torno a la 
se derivan del modo en que se conciben las orientaciones de distintas políticas públicas -en los ámbitos nacional e internacional- cuyos fines se contraponen para generar nuevas tensiones que en las instituciones se intentan resolver del mejor modo posible.

\section{Cambios normativos, estrategias y acciones para impulsar la educación inclusiva}

Teniendo en cuenta que para documentar esta experiencia se escogió el periodo 2014-2018 a continuación, se sintetizan las principales transformaciones y desarrollos en el ámbito normativo y en las prácticas y acciones generadas en esta coyuntura, para materializar sus políticas institucionales y consolidar su proyecto de educación inclusiva, en una universidad pública como la UPN de Colombia. Los distintos procesos se organizan en relación con ejes estructurales en torno a los cuales, sin embargo, es posible advertir que la universidad supera aquellas expectativas que se había formulado en torno al PDI vigente.

\subsection{Democratización del acceso a la educación superior}

Si bien subsiste como problemática en la universidad colombiana la presencia de diversos factores de discriminación, ya sea de clase, raza, sexo, género, o etnia, que generan en el acceso una mezcla entre mérito y privilegio, los esfuerzos de la Universidad en los últimos años han mostrado logros significativos, tanto en el plano nacional como en el institucional. En el primer caso, las IES del sector oficial representan el 28\% de las IES del país (81) con una tasa de participación en matrícula a 2018 equivalente al 50,8\% (1.241.790 estudiantes), mientras que el 49,2\% (1204524) corresponde al sector privado, que cuenta con el $72 \%$ de las instituciones del sector (211 IES en 2017). Para el caso de la UPN, en la matrícula de los últimos años (período 2014-2018), se ha presentado un promedio de 1335 estudiantes matriculados en primer semestre, en su mayoría de pregrado (80\% de la matrícula): en el año 2015 se presentó el promedio más alto (1 435 matriculados) y en 2014 el promedio más bajo (1250). En el número total de matriculados de pregrado y posgrado las cifras son similares: 9752 es el promedio de todo el período; 2015 es el año que registra el promedio más alto con 10022 estudiantes y el promedio más bajo corresponde al año 2017, con 9489 estudiantes.

Ahora bien, esta matricula es necesario analizarla más detalladamente en relación con los cambios normativos impulsados en este período, relacionados con varios procesos que se desarrollaron de manera simultánea y que buscaban favorecer y ampliar los mecanismos de selección, ingreso y admisión inclusiva de los aspirantes pertenecientes a grupos tradicionalmente excluidos en el acceso a la educación superior, así como mejorar los procesos de ingreso y admisión a la Universidad.

En el primer caso, se formularon dos normativas complementarias: una, para ampliar el alcance de la admisión inclusiva y contemplar, además de los aspirantes pertenecientes a poblaciones de grupos étnicos (en la norma anterior se estipulaba que estaba dirigido a miembros de comunidades indígenas, rom o gitano, negras, afrocolombianas, palenqueras y raizales), a las víctimas del conflicto armado en Colombia, desmovilizados en proceso de reintegración y habitantes de frontera. De esta forma, se buscó garantizar una educación superior inclusiva que disminuyera barreras de acceso y favoreciera la permanencia en la Universidad de poblaciones que requieren especial protección. Y la otra, se orientó a formalizar un proceso que llevaba casi dos décadas de desarrollo, pero sin una regulación que favo-

identificación de barreras para el aprendizaje y la participación, a fin de que sean las instituciones las que desarrollen adecuaciones (no solo de infraestructura y equipamientos) para lograr superarlas. Así, la educación inclusiva, tal como se aclara en la política nacional colombiana (Ministerio de Educación Nacional [MEN], 2013) trasciende la connotación de un objetivo concreto que busca el ingreso al sistema educativo, para concebirse como un proceso inacabado y de largo plazo, en el que tanto el Estado como las instituciones de educación superior se ven comprometidos. 
reciera tanto la admisión como la educación inclusiva para aspirantes sordos, con discapacidad visual y con discapacidad física-motora.

En el segundo caso, se produjo una reorganización de los procesos de selección y admisión, a partir de criterios de equidad, transparencia y racionalidad, así como el desarrollo de procedimientos ágiles y eficientes, según las particularidades que se generan en los programas y el número de aspirantes que se presentan en cada etapa, para mejorar los índices de selección y de absorción. En cada una de estas normas también se da importancia a las acciones de seguimiento y evaluación por parte de las unidades académicas, de tal manera que se logre la cualificación permanente de estos procesos de admisión y su articulación con la permanencia en la Universidad.

Los efectos de estas medidas los podemos apreciar en algunos datos relevantes: de una parte, se observa un significativo aumento en el porcentaje de estudiantes de pregrado seleccionados y matriculados a través del proceso de admisiones inclusivas, especialmente en los años 2017 y 2018, período en el que se duplica ampliamente el porcentaje de admisión en comparación con el año 2014. Este aumento es resultado de los cupos asignados para las poblaciones contempladas en la nueva normatividad interna, lo cual representa un promedio de matriculados por período de 560 estudiantes (ver Tabla I).

Tabla I. Admisiones inclusivas pregrado últimos cinco años

\begin{tabular}{lllll}
\hline & AÑO & $\begin{array}{c}\text { TOTAL ESTUDIANTES UPN } \\
\text { PREGRADO }\end{array}$ & $\begin{array}{c}\text { TOTAL ESTUDIANTES MATRI- } \\
\text { CULADOS POR ADMISIONES } \\
\text { INCLUSIVAS }\end{array}$ & $\begin{array}{c}\text { PORCENTAJE DE ADMISIONES } \\
\text { INCLUSIVAS EN LA UPN (\%) }\end{array}$ \\
\hline 2014 & 9840 & 381 & 3,87 \\
\hline 2015 & 9962 & 464 & 4,66 \\
\hline 2016 & 9664 & 493 & 5,10 \\
\hline 2017 & 9506 & 683 & 6,71 \\
\hline 2018 & 9796 & 823 & 8,40 \\
\hline Promedio & 9754 & 560 & 5,75 \\
\hline
\end{tabular}

Fuente: Subdirección de Admisiones y Registro, 2019

En este proceso, también es posible identificar las tendencias en torno a la admisión de los aspirantes sordos, con discapacidad visual y con discapacidad física-motora. Los datos de los últimos años reportados en la Tabla II, muestran los importantes avances para crear las condiciones de acceso a estos grupos poblacionales.

Tabla II. Matrículas a primer semestre, estudiantes sordos, con discapacidad visual y discapacidad físico-motora, período 2014-2018

\begin{tabular}{lcccc}
\hline \multirow{2}{*}{ AÑO } & \multicolumn{1}{c}{ MATRICULADOS PRIMER SEMESTRE } & \multicolumn{2}{c}{ TOTAL POR AÑO } \\
\cline { 2 - 5 } 2014 & Sordos & Con discapacidad visual & Con discapacidad físico-motora & 14 \\
\hline 2015 & 15 & 8 & 4 & 27 \\
\hline 2016 & 8 & 10 & 2 & 15 \\
\hline 2017 & 12 & 5 & 1 & 21 \\
\hline 2018 & 7 & 25 & 6 & 38 \\
\hline Total & 44 & 56 & 15 & 115 \\
\hline
\end{tabular}

Fuente: Subdirección de Admisiones y Registro, 2019 
De otra parte, en cuanto a los indicadores que permiten evaluar la selección, el acceso y la eficiencia en la educación superior, esto es, en qué porcentaje los inscritos son admitidos en la institución (selectividad) y cuántos de los admitidos se matriculan efectivamente (absorción), se aprecian igualmente cambios importantes, tal como se revela en los datos de la Tabla III.

Tabla III. Índices de selectividad y absorción en programas de pregrado UPN 2014-2018

\begin{tabular}{|c|c|c|c|}
\hline AÑO & PERÍODO & ÍNDICE DE SELECTIVIDAD (\%) & ÍNDICE DE ABSORCIÓN (\%) \\
\hline \multirow{2}{*}{2014} & I & 14 & 92 \\
\hline & $\|$ & 20 & 87 \\
\hline \multirow{2}{*}{2015} & 1 & 17 & 89 \\
\hline & $\|$ & 22 & 94 \\
\hline \multirow{2}{*}{2016} & I & 17 & 84 \\
\hline & $\|$ & 20 & 95 \\
\hline \multirow{2}{*}{2017} & I & 13 & 108 \\
\hline & $\|$ & 18 & 95 \\
\hline \multirow{2}{*}{2018} & I & 20 & 105 \\
\hline & $\|$ & 18 & 106 \\
\hline
\end{tabular}

Fuente: Subdirección de Admisiones y Registro, 2019

Respecto al índice de selectividad (relación entre admitidos/inscritos), el promedio para el período 2014-2018 fue del 18\% para los programas de pregrado y del 77\% para los de posgrado, tendencia ligada al hecho de que se presenta un mayor número de inscritos en los programas profesionales respecto a los de posgrado, y a la capacidad institucional para ampliar el acceso a la UPN, lo cual genera un alto nivel de selectividad en el pregrado: de cada 10 aspirantes, la universidad admite aproximadamente a dos. En cuanto al índice de absorción (relación entre matriculados a primer curso/admitidos) se destaca la tendencia creciente, e incluso en tres semestres se supera el 100\% para los programas de pregrado, con un promedio del período equivalente al $96 \%$, y del $86 \%$ para el caso de posgrado; datos que revelan una alta eficiencia institucional.

Esta tendencia resulta especialmente significativa al comparar los programas de la UPN con otras licenciaturas y con el conjunto de programas universitarios del país. Según los datos del MEN (2016) en el estudio de Arias, Díaz, Garzón, León, Rodríguez y Valbuena (2018) ${ }^{4}$, es posible identificar que en el período 2012-2015, el promedio de estos índices para los programas universitarios fue del 51,3\% en la selectividad y del 78,4\% en la absorción; mientras que en el caso de los programas de licenciatura los promedios fueron de 54,5\% y 86,1\%, respectivamente. Los datos de la UPN también controvierten la idea de que en los programas de formación inicial de maestros la selectividad es baja y se generan limitaciones en la absorción de quienes son admitidos. No obstante, esta alta selectividad para los programas también se constituye en una tensión frente a la expectativa de democratizar el acceso, pues la UPN no cuenta con las condiciones necesarias para responder a la amplia demanda que se presenta en sus programas de formación, particularmente en el nivel de pregrado.

Este conjunto de logros institucionales tuvo una repercusión externa, pues significó la entrega de la distinción a la Universidad Pedagógica Nacional por parte del Ministerio de Educación Nacional como Institución de Educación Superior Inclusiva, otorgado en diciembre de 2018.

4 Investigación desarrollada por seis grupos de investigación de la UPN y financiada por Colciencias 


\subsection{Fomento y estímulo a la permanencia y la graduación}

En relación con los procesos vinculados a favorecer el acceso y a estimular la permanencia y la graduación en la Universidad de todos los estudiantes, no solamente de aquellos que se consideran en «riesgo de deserción» o de quienes han ingresado en las modalidades de educación inclusiva, es posible identificar un conjunto de acciones que se traducen en logros importantes y se podrían catalogar como la coexistencia entre alternativas compensatorias para estimular la retención o atender parcialmente problemáticas sociales -concepción asistencial-, y medidas que se preocupan por favorecer la participación, la convivencia, los procesos de autorregulación y consolidar alternativas de bienestar universitario, que responden a una visión integradora en torno a proyectos colectivos de lo que se plantea como la búsqueda del buen vivir y el afianzamiento de valores comunitarios.

Un primer aspecto que se reorganizó institucionalmente fue el Grupo de Orientación y Acompañamiento Estudiantil (GOAE), instancia formalizada a través de la Resolución rectoral 0308 del 11 de marzo de 2016, adscrita a la Vicerrectoría Académica, la cual se estructuró para favorecer tanto la admisión, como la creación e implementación de estrategias que posibilitaran el acompañamiento académico, la permanencia y graduación de los estudiantes en la UPN, basado en un enfoque diferencial y de educación inclusiva.

Esta instancia se responsabilizó de proyectos de inversión como el denominado Educación inclusiva, el cual se orientó a diseñar y desarrollar estrategias que favorecieran la inclusión de los estudiantes. En esta misma línea, se organizó el Seminario Taller Vida Universitaria UPN, cuyo propósito consistió en articular las propuestas dirigidas al fortalecimiento de las políticas de inclusión, disminuir la deserción y favorecer la convivencia universitaria, basada en el respeto a los derechos humanos. Asimismo, se implementó el Centro de tutorías, cuyo propósito se dirigió a fomentar la calidad educativa y la permanencia mediante la atención personalizada frente a las problemáticas que influyen en el desempeño académico de los estudiantes; igualmente, generar un espacio de formación y reflexión que posibilitara a los estudiantes tutores, adquirir y generar estrategias pedagógicas y didácticas que potenciaran su experiencia como docentes.

Para favorecer los procesos de inclusión de grupos excluidos por sus condiciones socioeconómicas se destacan, además, las acciones realizadas durante los años 2017 y 2018, orientadas a formular un convenio con instituciones del sector oficial para ofrecer incentivos económicos y condiciones psicosociales que contribuyeran al sostenimiento de la población matriculada en los programas de pregrado, pertenecientes a la zona de influencia del relleno sanitario Doña Juana. Esta es una zona que, destinada al depósito de basuras sin las medidas adecuadas, generó damnificados que hoy el Distrito de Bogotá debe indemnizar. Precisamente, gracias a dichos convenios, fue posible brindar apoyo a 213 estudiantes de las diferentes licenciaturas que habitan en la localidad de Usme o en las veredas de la localidad de Ciudad Bolívar ${ }^{5}$, con un auxilio económico que les permitió su sostenimiento.

Respecto a la orientación y acompañamiento para evitar la deserción, las iniciativas propuestas en la perspectiva psicosocial se adelantaron en dos ámbitos: desde el GOAE y en Bienestar Universitario, donde se continuaron las tareas del programa psicosocial que permitieron superar cada vez más la atención y los servicios prestados a la comunidad universitaria.

5 Según la Universidad del Rosario (2009), estas localidades de la ciudad de Bogotá se caracterizan por la confluencia de múltiples problemáticas, pues concentran dificultades sociales y económicas, con una precaria presencia de las entidades estatales. Ciudad Bolívar, por ejemplo, es la zona de la capital con mayor cantidad de población desplazada (26\% del total), y aunque la mayoría de los habitantes están clasificados en estratos socio-económicos 1 y 2 , el $17 \%$ se sitúa por debajo de la línea de miseria. 
La institución cuenta igualmente con programas de apoyo socioeconómico que contribuyen a minimizar las condiciones socioeconómicas que pueden afectar la permanencia de los estudiantes en la Universidad, para disminuir de esta forma los índices de deserción generados por esta situación. Con este propósito se ofrece el servicio de restaurante y cafetería subsidiados, gracias a lo cual los estudiantes pueden adquirir desayuno y almuerzo balanceado y nutritivo por solo un tercio de dólar, productos de cafetería a precio de costo, y en ocasiones cena o refrigerio vespertino, por una módica suma.

Al lado de los beneficios anteriores, la Universidad cuenta con diversos apoyos socioeconómicos, entre los cuales figuran: a) Reliquidación de matrícula, para establecer matrículas mínimas y justas para los y las estudiantes, b) Fraccionamiento de matrícula, para facilitar su pago cuotas; c) monitorias ASE, que entrega dos salarios mínimos mensuales ${ }^{6}$ a los y las estudiantes para apoyarlos en su manutención.

Tabla IV. Programa de apoyo socioeconómico 2014-2018

\begin{tabular}{lrrrrrrr}
\hline \multicolumn{1}{c}{ PROGRAMA } & 2014 & 2015 & 2016 & 2017 & 2018 & TOTAL \\
\hline Reliquidación de Matrícula & 359 & 250 & 171 & 202 & 95 & 1077 \\
\hline Fraccionamiento de Matrícula & 17 & 11 & 15 & 134 & 141 & 318 \\
\hline Monitorias ASE & 163 & 165 & 157 & 154 & 112 & 751 \\
\hline Restaurante* & 4497 & 4797 & 5164 & 5446 & 2707 & 22611 \\
\hline
\end{tabular}

Fuente: Subdirección de Admisiones y Registro, 2019

* Nota: Los datos corresponden a número de estudiantes beneficiados.

Los datos en torno a este tipo de apoyo para el período 2014-2018 muestran el crecimiento sostenido en todas las alternativas ofrecidas por la universidad (ver Tabla IV). En este periodo, en promedio, 1238 estudiantes por año fueron beneficiados con algún tipo de apoyo socioeconómico. Es decir que alrededor del 13\% de los estudiantes de pregrado y posgrado de la universidad han contado con beneficios relacionados con los programas de apoyo socioeconómico. Así mismo, en este período se generó un importante incremento en los apoyos económicos a través de: monitorias académicas (de 98 a 109), monitorias de investigación (767 estudiantes entre 2014 y 2018) y semilleros (98 estudiantes articulados a 15 semilleros). Se incrementaron igualmente los apoyos para asistencia a eventos internacionales y se ofreció, por primera vez, financiación total, incluidos costos de desplazamiento e instalación para realización de semestres académicos en instituciones universitarias del exterior.

Otras acciones que lograron afectar positivamente, no solo la permanencia de los estudiantes, sino generar procesos complementarios en su desarrollo profesional e incentivar el sentido de pertenencia, fueron los estímulos y reconocimientos que se otorgaron en retribución a la excelencia académica, y los logros destacados a nivel cultural y deportivo. Estos incentivos son: becas de pregrado y posgrado; matrículas de honor; monitorias: de docencia, investigación, extensión y gestión institucional; participación en el grupo estudiantil de protocolo institucional; y, semilleros de investigación. Todas ellas cuentan con mecanismos normativos que favorecen su implementación basada en criterios de equidad y excelencia, y estrategias de divulgación para promover sus posibilidades de acceso. En la Tabla V se muestran los datos para este período, en la que se destacan los logros acumulados para algunos de estos estímulos (becas, matrículas de honor y becas gestionadas con la Secretaría de Educación del Distrito -SED-): en total se otorgaron 1032, de las cuales el mayor número se otorgó entre los años 2015 a 2017.

6 Un salario mínimo mensual legal vigente (SMMLV) en Colombia representa alrededor de 262 USD. 
Tabla V. Estímulos, becas, matrícula de honor, otorgadas en el período 2014-2018

\begin{tabular}{|c|c|c|c|}
\hline AÑO & PROGRAMA DE BECAS & MATRÍCULA DE HONOR & BECAS SED \\
\hline 2014 & 33 & 71 & - \\
\hline 2015 & 30 & 80 & 129 \\
\hline 2016 & 30 & 80 & 190 \\
\hline 2017 & 32 & 75 & 127 \\
\hline 2018 & 19 & 39 & 97 \\
\hline TOTAL & 144 & 345 & 543 \\
\hline
\end{tabular}

Fuente: Subdirección de Admisiones y Registro, 2019

En buena medida, esta multiplicidad de acciones lograron repercutir en las tasas de deserción anual y en la retención de los estudiantes de la UPN pues, tal como se aprecia en la gráfica 1, a partir del año 2015, la tasa de deserción anual, esto es, la proporción de estudiantes que estando matriculados dos semestres atrás son clasificados como desertores un año después ${ }^{7}$, ha disminuido de manera significativa: entre 2014 y 2015 la tasa disminuyó más de la mitad, pasando del 18\% al 8,4\% y se ha mantenido en un promedio del 9,04\%; consecuentemente, la tasa de retención se ha incrementado con un promedio del 90,96\% entre 2015 y 2018.

\section{Gráfico 1. Tasa de deserción anual UPN período 2014-1 - 2018-2}

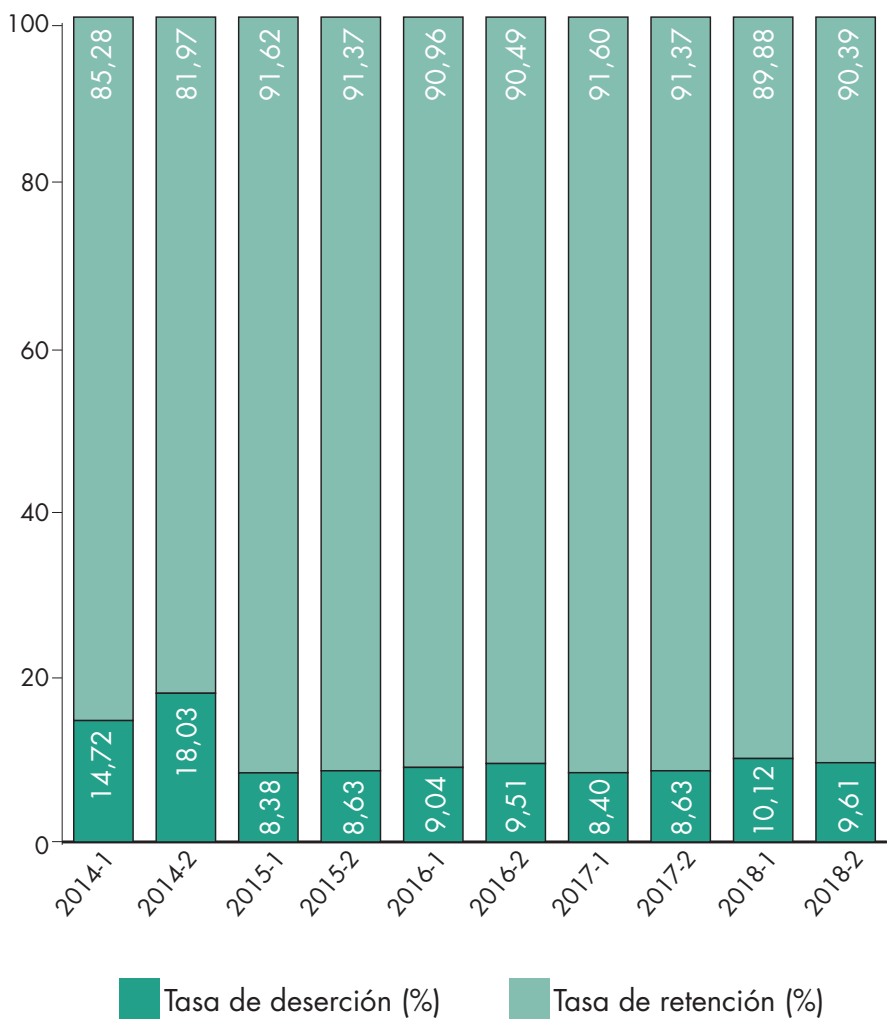

Fuente: SPADIES 3.0. Fecha de consulta: 26/03/2019.

También se destaca que en los años 2015 y 2017 se presentaron las tasas de deserción anual más bajas y, por tanto, las tasas de retención más altas (por encima del 91\%). Así mismo, al comparar estos

Es importante aclarar que el concepto de deserción que maneja el sistema SPADIES del Ministerio de Educación Nacional es diferente al que maneja la institución, pues en la UPN un estudiante puede solicitar reintegro luego de dos años (cuatro semestres) de ausencia. 
datos con la tasa de deserción anual universitaria a nivel nacional, el dato también es favorable, pues para el año 2015 (MEN, 2016: 296) esta tasa fue del 9,25\%, ubicándose la UPN un punto por debajo de este valor.

En torno a la promoción y graduación, se destaca el proceso de Amnistía aprobado por el Consejo Académico en el año 2015, el cual propició una nueva admisión de personas que perdieron la calidad de estudiantes por circunstancias diferentes a la expulsión de la Universidad, cuando les faltaba cursar y aprobar como máximo el $25 \%$ de los créditos requeridos para obtener el título. De los 118 admitidos en este proceso para los programas de pregrado, se registra un $62 \%$ de graduados, y de los 30 admitidos a programas de posgrado se graduó el 37\%, según el informe de gestión de la rectoría del período 2014-2018 (UPN, 2018). Adicionalmente, y como contribución a los procesos de graduación, se han realizado procesos de acompañamiento a proyectos de grado de estudiantes con discapacidad que requieran apoyos específicos relacionados con los programas y servicios que ofrece el GOAE.

A nivel global, las cifras de graduación muestran que en los años 2014 y 2018 se generó el mayor número de graduados tanto de pregrado como de posgrado, para un total general de 9.184 graduados, de los cuales el $71 \%$ corresponde a programas de pregrado y el $29 \%$ de posgrado.

\subsection{Apertura de la participación en el gobierno universitario y en la construcción de políticas institucionales}

A partir del año 2014, la nueva administración de la Universidad invitó a la comunidad a involucrarse de manera concreta en la construcción colectiva del gobierno institucional y sus políticas. El propósito era impulsar la transformación de la realidad universitaria, desde múltiples sentidos, procesos y prácticas. En esta dirección, en el segundo semestre de ese mismo año se emprendieron las siguientes acciones:

- Ponte la 10 por la UPN. Con esta campaña se logró que estudiantes, trabajadores, funcionarios y profesores, pintaran y enlucieran la Universidad en sus distintas sedes y, principalmente, en la central ubicada en la Calle 72 de Bogotá. Cerca de diez años de abandono y desidia, lugares destruidos por los conflictos entre fuerza pública y estudiantes, quedaron atrás gracias a la convocatoria respondida en toda una semana de trabajo.

- Elaboración participativa del Plan de Desarrollo Institucional (PDI). Paralelo a las jornadas de pintura y enlucimiento de la Universidad, con una metodología previamente discutida con grupos estudiantiles y profesorales, se empezó a trabajar y concretar la elaboración colectiva del PDI. Por primera vez en la Universidad, se propuso de manera amplia y abierta una discusión de compromiso y futuro, que ganó la voluntad de muchos miembros de la comunidad.

- Diálogos por la pazy la convivencia. Con esta iniciativa se invitó, en primer lugar, a todos los colectivos clandestinos de la Universidad para que expresaran abiertamente su pensamiento y se brindó la posibilidad de aperturas para la confianza, la convivencia y la democracia. Ello permitió minimizar los disturbios en la Universidad y reducir la existencia de grupos con dicha naturaleza. De un promedio de 14 disturbios al semestre, se redujo su cantidad a 2, causados ahora por motivos externos a la Universidad y ajenos a sus políticas y decisiones de su dirección.

- Vigilia pedagógica. Al promover el sentido de pertenencia, se logró la expresión estudiantil para obtener una estampilla distrital que, como tributo entregado a la Universidad, recaudara recursos para la 
edificación de una nueva sede universitaria. Después de casi cuatro años en busca de su reglamentación por el Concejo de Bogotá, se obtuvo el cometido gracias a la movilización y sensibilización del ente distrital.

- Semana Universitaria de las Culturas. Con ello se institucionalizó una semana de encuentro universitario de poesía, música, teatro, arte, literatura y demás formas de expresión cultural sin límite y con pleno apoyo por parte de la academia universitaria.

- Memorial a las víctimas de la UPN. Por primera vez se hizo un reconocimiento a las víctimas del conflicto armado en Colombia. Se otorgaron títulos póstumos a estudiantes caídos con motivo del conflicto, se les rindió homenaje con sus familias y con participación abierta, se construyó un mural en el edificio administrativo, que incluyó a trabajadores y egresados, como tributo a su recuerdo y permanente presencia en la vida universitaria.

\subsection{Ampliación de los alcances del bienestar universitario}

En una universidad con estudiantes de estrato social 1 y $2^{8}$, ofrecer servicio de almuerzo y cafetería subsidiados es una necesidad indiscutible. De esta manera, en 2014 se atendían 4497 usuarios en servicio de almuerzo, que pasaron en 2017 a 5446; es decir, 949 usuarios más por año. La cafetería de la sede Calle 72, que cumplía cerca de un año cerrada, se abrió de nuevo para brindar desayuno subsidiado y productos alimenticios a precio de costo durante todo el día e incluso los sábados. Igualmente, se asumió el servicio en la Escuela maternal, se abrió tanto el restaurante en la sede Valmaría para la Facultad de Educación Física, como las cafeterías en las sedes de Parque Nacional y Nogal, para la Facultad de Artes.

Labor importante fue también dignificar el trabajo de los trabajadores dotándolos de la indumentaria adecuada, del equipo de cocina y del menaje de producción y servicio pertinentes. Se vincularon ingeniera de alimentos, nutricionista y cocineros profesionales en procesos de selección por méritos académicos y experiencia, y se apoyó a trabajadores para cursar estudios en gastronomía. Se abrió, además, el servicio de panadería y frutería en la sede principal y se ofreció refrigerio vespertino subsidiado y cena en las oportunidades en que la biblioteca funcionó 24 horas. En todas las sedes se ofertó la posibilidad de almuerzo vegetariano para los estudiantes que así lo prefirieran.

En relación con la salud, se dio un salto importante: se adecuaron los consultorios médicos y odontológicos de la sede central y se abrieron nuevos sitios de atención médica, de enfermería y fisioterapia en la sede Valmaría. Esto permitió pasar de 16097 consultas anuales en 2014 a 21645 en 2017. Así mismo, se ofertó un nuevo servicio médico en promoción y prevención para atender enfermedades de transmisión sexual ETS, salas amigas de la familia lactante, prevención de fisioterapia, cuidado corporal, calistenia, higiene postural, planificación familiar y riesgo cardiovascular que, de 8673 atenciones en su primer año (2015), pasó a 10532 en 2017.

Con el propósito de ofrecer actividades alternas y extracurriculares a los y las estudiantes, se implementó el Programa Cultural a través de tres ejes: la formación integral, la cultura como escenario para la convivencia, y la circulación de trabajos artísticos. Mediante una variada oferta de talleres en artes y humanidades dirigidos a la comunidad universitaria, se pasó de solo 10 en el año 2014, a 18 en 2017.

\footnotetext{
Según el Departamento Administrativo Nacional de Estadística (DANE), el estrato socioeconómico en Colombia es determinado por el lugar en donde viven los usuarios. Aquellos con menores recursos, situados en barrios periféricos y con viviendas de interés social y grandes necesidades y limitaciones, son clasificados en los estratos 1 y 2 .
} 
Por otra parte, el deporte y la recreación se proyectaron como espacio de crecimiento personal y colectivo para fortalecer el sentido de pertenencia. En esta dirección, por primera vez se dio apoyo a las iniciativas estudiantiles que, mediante el deporte y la recreación, generaran procesos comunitarios en pro de la convivencia y el fortalecimiento de la identidad. Así mismo, se fortaleció el deporte representativo de la comunidad universitaria, y se amplió la participación en eventos regionales y nacionales: de 1029 actividades deportivas anuales en 2014, se pasó a 2772 en 2017, mucho más del doble. Una de sus deportistas, incluso, fue seleccionada para los Juegos Olímpicos de Río de Janeiro en 2016. La UPN, que se había aislado de la máxima competencia deportiva universitaria nacional, volvió a ella: los juegos de la Asociación Colombiana de Universidades (ASCUN) en 2017, y obtuvo participación en el cuadro de medallas.

\subsection{Atención a problemas socialmente relevantes}

Con apoyo de redes externas e instituciones estatales, la Universidad implementó jornadas de atención mediante la estrategia de Acogida para jóvenes, con el fin de identificar riesgos en salud mental, salud sexual y reproductiva y condiciones crónicas en los espacios comunitarios, a partir del tamizaje en VIH, Sífilis y APGAR (examen practicado a recién nacidos).

Además, se abordaron problemáticas relacionadas con el consumo de sustancias psicoactivas a través de escucha activa, la identificación temprana de riesgos en salud asociados al consumo, intervenciones breves a través de entrevista motivacional, y la remisión médica según las necesidades de la población.

Durante el período 2014-2018 se construyeron las Rutas para la atención en salud mental, la Ruta para atención y prevención de eventos suicidas y, mediante un Estudio sobre convivencia y violencias de género en la UPN, se sentaron las bases para la redacción de los protocolos para la atención y prevención de las violencias contra las estudiantes de la UPN, para la atención a eventos suicidas y para la atención a crisis por consumo de drogas.

\subsection{Los avances en la inclusión en los procesos académicos}

Para el caso de procesos académicos que favorecen la inclusión, desde los que se propone definir currículos flexibles y con perspectiva interdisciplinar contar con didácticas innovadoras y servicios de apoyo pedagógico para tutorías y nivelaciones según se requiera, y en los que se contempla el uso de las TIC, tal como se formula en los llamados «retos ${ }^{9}$ de la educación superior inclusiva» (MEN, 2013: 51-58), es necesario destacar la presencia desde 2003 del proyecto Manos y Pensamiento: inclusión de estudiantes sordos a la vida universitaria, desde el cual se ha impulsado la inclusión integral de la población sorda en los diferentes programas de pregrado de la Universidad, y cuyo propósito central es la formación de licenciados sordos para atender las necesidades educativas de la población sorda colombiana. A partir del estudio, la investigación, la reflexión y el debate permanentes sobre el sujeto sordo y sus necesidades de desarrollo educativo, social y laboral, se ha ido construyendo un modelo de inclusión para atender las necesidades específicas de dicha población, que también supone una interacción mediada lingüísticamente (intérpretes de lengua de señas). Estas interacciones representan una posibilidad de hacer partícipes de la educación inclusiva a los profesores de la UPN y de promover un cambio de

\footnotetext{
Los retos planteados son: desarrollar procesos académicos inclusivos, contar con profesores inclusivos, promover espacios de investigación e innovación y creación artística y cultural con enfoque de educación inclusiva, construir una infraestructura administrativa y financiera que sustente las estrategias y acciones en este campo, y, diseñar una política institucional de educación inclusiva que sustente los demás retos (MEN, 2013).
} 
mentalidad general sobre su rol, que implica también el desarrollo de procesos de formación permanente respecto a prácticas pedagógicas articuladas a la diversidad y particularidad de los estudiantes.

Otro ámbito de desarrollo institucional en este campo está representado en la producción de conocimiento en y para la educación inclusiva a través de la investigación generada por los grupos de investigación de la Universidad. En esta dirección se destacan las investigaciones desde la Facultad de Educación, que desde el año 2010 a la fecha cuenta con 14 proyectos que apuntan a la construcción colectiva de conocimiento en relación con la educación inclusiva, tanto hacia el interior de la Universidad como en el contexto nacional. Desde estos proyectos se estimula la participación de los estudiantes de la UPN en el rol de monitores, a través de un semillero que promueve procesos de formación en este campo.

\section{Tensiones y logros en el desarrollo de la educación inclusiva en la Educación Superior}

Para Universidades públicas como la UPN, promover y llevar a cabo una política integral y flexible de educación superior inclusiva (ESI) desde un enfoque diferencial, significa un reto exigente, en especial por las múltiples transformaciones que representa la concreción de las acciones y estrategias estipuladas desde la política educativa estatal, sin contar con las condiciones y los recursos que hagan posible todos los cambios esperados.

Según se concibe desde los Lineamientos de la Política de Educación Superior Inclusiva, formulados por el Ministerio de Educación Nacional (2013), este enfoque pretende no solamente hacer visibles las formas de exclusión que se generan para el acceso y permanencia en este nivel educativo, sino también identificar barreras para el aprendizaje y la participación, de tal modo que se logre superarlas. Esto implica, se dice, asumir la educación inclusiva como un proceso inacabado y de largo plazo, en el que el Estado y las instituciones de educación superior se comprometen con sus diversos logros. Para sustentar esta expectativa, el discurso de la política oficial retoma formulaciones de la $48^{\text {ava }}$ reunión de la Conferencia Internacional de Educación (CIE), en términos de la necesidad de impulsar

«politicas intersectoriales, con un enfoque de derechos, que aborden de forma integral los factores que generan exclusión dentro y fuera de los sistemas educativos; combinar estrategias globales para toda la población, enfatizando las de carácter preventivo, con medidas focalizadas para los colectivos en situación de mayor vulnerabilidad; avanzar desde estrategias homogéneas hacia políticas que consideren la diversidad con cohesión social, y el desarrollo de curriculos, y sistemas de evaluación inclusivos» (Blanco, 2008: 14).

En consonancia con estas aspiraciones, el MEN (2013) desde los lineamientos, plantea acciones estratégicas para cada uno de los grupos priorizados ${ }^{10}$ en relación con el acceso, la permanencia, la graduación, y sobre la pertinencia y la calidad, a partir de la enunciación de las barreras en cada uno de estos aspectos y estipula los retos antes presentados que deben atender las IES para el desarrollo de la educación inclusiva. Adicionalmente, en torno a la pertinencia, se prevé la incorporación de criterios de educación inclusiva y accesibilidad en el Sistema de Aseguramiento de la Calidad en la Educación Superior, frente al cual las IES deben responder para la renovación de los registros calificados y la acreditación de sus programas de formación.

10 Los grupos priorizados por el MEN (2013) para la ESI son: Población estudiantil en situación de discapacidad y con capacidades y/o talentos excepcionales; grupos étnicos: comunidades negras, afrocolombianas, raizales y palenqueras, pueblos indígenas, pueblos Rrom, población víctima del conflicto armado en Colombia, población desmovilizada en proceso de reintegración y población habitante de frontera. 
De esta manera, por un lado, no se contemplan las implicaciones que para las IES públicas conllevaría el desarrollo de todas estas iniciativas diferenciales relacionadas con la implementación de la educación inclusiva, para las cuales se requiere contar con recursos que permitan adelantar cambios en: infraestructura, plantas de personal administrativo, aumento en el tiempos de los profesores, programas especiales de desarrollo profesoral, sistemas tecnológicos robustos y apoyos académicos diferenciados, proyectos y semilleros de investigación, creación de observatorios laborales, inexistentes hoy en la mayoría de las IES nacionales y regionales; así como una decisiva articulación con distintas entidades del orden territorial y nacional.

Estas pretensiones contrastan con las condiciones críticas de financiamiento de la educación superior pública que recientemente ocasionaron a un paro de más de cuatro meses por parte de las comunidades educativas de todo el país, ante la agobiante situación que data de 1992: con un incremento al presupuesto general de las IES equivalente al IPC anual y que dejaba por fuera, no solo el crecimiento real de las nóminas, sino la actualización de las IES, su modernización, el cuidado de sus plantas físicas, los avances tecnológicos y las mismas exigencias que el Estado genera, especialmente ligadas a la acreditación obligatoria de los programas de licenciatura y a la acreditación institucional.

Por otro lado, la política aboga por trascender el enfoque poblacional que se centra en la atención a grupos específicos de estudiantes para optar por un enfoque diferencial que implica privilegiar una educación para todos (EPT), en la que se contemplen los contrastes entre lo urbano y lo rural, los efectos de la privación sociocultural y el enfoque de género, sin dejar de lado la preocupación por las relaciones intersubjetivas entre los estudiantes. Es decir, que se promueven políticas diferenciales que potencien y valoren su diversidad, al mismo tiempo que protegen y defienden sus particularidades. Sin embargo, en la operativización de la política, nuevamente se opta por formular acciones estratégicas direccionadas para cada uno de los grupos identificados y priorizados. Esta misma tensión es la que experimenta la UPN y que intenta resolver al no desconocer la necesidad de realizar acciones direccionadas a atender las particularidades y especificidades de los diferentes grupos y de las barreras que se generan para su acceso y permanencia en la educación superior, pero a la vez, contempla la realización del proyecto formativo que se ha trazado con la totalidad de sus estudiantes.

Es decir, para las IES públicas, el desarrollo de acciones en torno a la educación inclusiva como se plantean desde el MEN (2013), con recursos tan limitados aún para llevar a cabo sus procesos misionales de docencia, investigación y proyección social de manera ordinaria, suponen una contradicción de la política basada en la EPT, pues si además se pretende establecer indicadores de existencia y cumplimiento de la educación inclusiva de las IES en el sistema de aseguramiento de la calidad, se verán abocadas a una mayor desfinanciación que afecta tanto el acceso, como la permanencia y la graduación, no solo de los grupos priorizados sino de todos los estudiantes, y a seguir buscando estabilidad a través de la búsqueda por recursos propios. Cabe entonces la pregunta por la necesaria corresponsabilidad que se requiere para alcanzar la ESI y por las condiciones que se crean para que efectivamente se logren impulsar políticas y acciones intersectoriales.

Otro aspecto que configura una tensión en las dinámicas institucionales, particularmente para las instituciones formadoras de maestros, se relaciona con otras políticas que se movilizan en el contexto nacional y que cuentan con el respaldo de las formulaciones de los organismos internacionales, relacionadas con propósitos que podrían considerarse contradictorios. Nos referimos a las formulaciones relacionadas 
con las políticas de educación inclusiva y las de mejoramiento de la calidad, que paradójicamente se plantean como articulables, a pesar de que en la práctica no necesariamente son compatibles.

Se trata de las exigencias que en la última década se han centrado en considerar al maestro como la influencia más determinante en el aprendizaje de los estudiantes y por tanto un factor clave para mejorar la calidad de la educación (Unesco, 2015; OCDE, 2005/2009; Banco Mundial, 2005, 2014, 2015; García, Maldonado, Perry, Rodríguez, y Saavedra, 2014). Paradójicamente, la relevancia de este actor se traduce en elevar las exigencias en todos los niveles para lograr «atraer personas con un nivel educativo elevado, conocimientos y potencial para convertirse en docentes eficaces» (Unesco, 2015: 21). Esta selectividad en el reclutamiento de los profesores conlleva a adoptar mecanismos como elevar los parámetros para el ingreso a los programas de formación docente, elevar la calidad de las instituciones de formación docente y elevar los estándares de contratación de los nuevos profesores (Banco Mundial, 2014-2015).

En Colombia estas orientaciones también se formularon en las Bases del Plan Nacional de Desarrollo 2014-2018, a partir de la definición de unas estrategias que buscaron y lograron incidir, por lo menos parcialmente en la ruta de la docencia desde la formación inicial hasta las condiciones de retiro de los profesores. En el tema que nos ocupa se formuló la «Atracción (otorgamiento de becas, créditos condonables y otros apoyos para que los mejores bachilleres del país ingresen a los programas de licenciatura), [así como] la optimización del proceso de ingreso a la carrera docente» (DNP, 2014: 43). Esta ruta se fundamentó en los cinco ejes estratégicos de la «propuesta sistémica de reforma para Colombia», exhaustivamente elaborada por el grupo de economistas contratados por la Fundación Compartir (García, et al., 2014).

En este contexto, nos encontramos con una tensión irresoluble entre las exigencias de las políticas que demandan generar mayores niveles en la calidad de la formación de los estudiantes y el adoptar la visión de la educación superior inclusiva que promueve la visibilización y el respeto por la diversidad; las universidades se encuentran hoy ante la imposibilidad, por un lado, de abrir el acceso a grupos históricamente excluidos y lograr una educación para todos y, por el otro, seleccionar a los mejores candidatos.

Por otra parte, llama la atención que se plantee que este tipo de selectividad para la formación inicial de los docentes se basa en principios de inclusión y equidad, de tal modo que los docentes en formación aprendan métodos de enseñanza que incluyan a todos los educandos y que les permitan conocer los mecanismos de exclusión, prejuicios y discriminación que operan en las instituciones educativas. No obstante, a las instituciones formadoras de maestros se les impele a atraer y seleccionar a los mejores, de tal modo que se opere con principios meritocráticos, es decir, con dirección a los pocos estudiantes que se destacan, lo que en últimas promueve la inequidad al no reconocer la perspectiva de una educación para todos.

Así mismo, se plantea que para lograr la eficacia del perfeccionamiento profesional de los docentes, de modo que se logre responder a las diversas comunidades de estudiantes, se ha de aplicar «un protocolo de codificación estándar a los planes de estudio de las instituciones de formación de docentes» (Unesco, 2016: 50); no obstante, frente a los postulados tanto de una educación para todos, como las políticas que abogan por la inclusión educativa y que promueven el desarrollo de programas flexibles, es contradictorio plantear la necesidad de estandarizar los planes de estudio. Igualmente, se señala la importancia de reconocer las particularidades y la diversidad de los contextos institucional, nacional y regional, y evitar la uniformidad, pero al mismo tiempo se demanda, en aras de mejorar la calidad, la necesidad de promover normas comparativas. 


\section{Conclusiones}

Como hemos visto, las condiciones de posibilidad de las políticas institucionales, que en este caso han logrado múltiples desarrollos en torno a lo que se ha denominado Educación Superior Inclusiva, atraviesan por diversas y exigentes tensiones que se sintetizan en tres grandes problemáticas, algunas de ellas interrelacionadas. La primera se refiere a impulsar una política integral y flexible de educación inclusiva, no solamente para aquellos grupos tradicionalmente excluidos del sistema educativo -en este caso de la educación superior- bajo las enormes restricciones económicas en que operan las IES estatales en Colombia y en consonancia con los requerimientos que los lineamientos de política le formulan, especialmente a las IES del sector oficial. La segunda radica en la coexistencia de exigencias que se orientan en direcciones opuestas y por tanto, en la práctica resultan contradictorias: de una parte, las formulaciones de la ESI que promulga una educación no discriminatoria y que favorezca la EPT, y las políticas de calidad que han optado por centrar las reformas y exigencias en elevar los parámetros para el ingreso a los programas de formación docente o generar estímulos solo a los mejores, así como incrementar los controles y las exigencias de calidad de las instituciones de formación de maestros. En la práctica, estas políticas se traducen en mayores niveles de selectividad y exclusión de aquellos que se considera que no cumplen con los estándares, incluidos los mismos programas, pues también se plantea forzar su cierre cuando no se cumplen los estándares esperados. La tercera consiste en la tensión estructural a la que asistimos en la educación superior en Colombia cuando el papel del Estado se concentra en constituirse en instancia clave para lograr una mayor eficiencia del sistema, a partir de la supervisión activa, la evaluación y la rendición de cuentas, lo cual supone la formulación de fines e indicadores. Para ello, centra su acción en responsabilizar a los sujetos y a las instituciones, incluidas las del propio sector estatal, de alcanzar las metas, pero también de la consecución de los recursos para aquello con lo que el mismo Estado no se logra comprometer.

Pese a estas tensiones, en la perspectiva de la ESI, la UPN avanza en el desarrollo de los retos propuestos desde el MEN en términos de los principios de flexibilidad e integralidad, no como una imposición desde la política y con interés de rendición de cuentas, sino desde la autonomía y principios sobre los que basa la esencia de su misión.

Igualmente, la UPN dispone y organiza sus unidades administrativas que sustentan la organización y orientación de las estrategias y acciones que promueven la ESI, de tal manera que no sea un compromiso solamente de instancias como Bienestar universitario, sino que se reconocen como transversales.

Como se ha mostrado, la UPN desarrolla un trayecto en el que, a pesar de las dificultades, se propuso establecer adecuaciones estructurales (normativas, de formación, de bienestar, de desarrollo docente, de atención a problemas relevantes, de participación e identidad institucional) que, con responsabilidad y compromiso, le permitan seguir trabajando por ser una universidad incluyente para todas y todos los estudiantes y no solo para los grupos considerados desde la política, como poblacionales vulnerables.

\section{Referencias bibliográficas}

Hernán Arias Gómez, Diego; Cecilia Díaz Flórez, Olga; Garzón Barragán, Isabel; León Palencia, Ana Cristina; Rodríguez Ávila, Sandra Patricia; Orlay Valbuena Ussa, Édgar (2018). Entre las exigencias de calidad y las condiciones de desigualdad: formación inicial de profesores en Colombia. Bogotá: Universidad Pedagógica Nacional, Colciencias. http://dx.doi.org/10.17227/ecp.2018.6581, consultado el 10 de febrero de 2019. 
Blanco, Rosa (2008): "Haciendo efectivo el derecho a una educación de calidad sin exclusiones". Revista Colombiana de Educación, 54, 14-35. http://revistas.pedagogica.edu.co/index.php/RCE/article/ view/5274/4307, consultado el 8 de abril de 2019.

Banco Mundial (2005). Mejorar la enseñanza y el aprendizaje por medio de incentivos. ¿Qué lecciones nos entregan las reformas educativas de América Latina? Washington, D.C.: Banco Mundial. Documento elaborado por Vegas, Emiliana y Umansky, Ilana. http://documents.worldbank.org/curated/en/640681468045232759/ pdf/332660S.pdf, consultado el 15 de marzo de 2019.

Banco Mundial (2014). Resumen. Profesores excelentes. Cómo mejorar el aprendizaje en América Latina y el Caribe. Washington D.C.: Grupo del Banco Mundial. Documento elaborado por Bruns, Barbara y Luque, Javier. https://openknowledge.worldbank.org/bitstream/handle/10986/20488/Spanish-excellentteachers-report.pdf? sequence $=5$, consultado el 13 de febrero de 2019.

Banco Mundial (2015). Profesores excelentes. Cómo mejorar el aprendizaje en América Latina y el Caribe. Washington D.C.: Grupo del Banco Mundial. Documento elaborado por Bruns, Barbara y Luque, Javier. https://doi.org/10.1596/978-1-4648-0151-8, consultado el 28 de febrero de 2019.

Departamento Administrativo Nacional de Estadística [DANE]. Estratificación socioeconómica para servicios públicos domiciliarios [Página web]. https://www.dane.gov.co/index.php/servicios-al-ciudadano/servicios-informacion/estratificacion-socioeconomica, consultado el 13 de abril de 2019.

Departamento Nacional de Planeación [DNP] (2014). Bases del Plan Nacional de Desarrollo 2014-2018. Todos por un nuevo país. Bogotá: Imprenta Nacional de Colombia.

García, Sandra; Maldonado, Darío; Perry, Guillermo; Rodríguez, Catherine y Saavedra, Juan (2014). Tras la excelencia docente. Cómo mejorar la calidad de la educación para todos los colombianos. Bogotá: Fundación Compartir. https://www.fundacioncompartir.org/pdf/Tras $\% 201 \mathrm{a} \% 20$ excelencia $\% 20$ docente $\% 20-\% 20$ estudio\%20final.pdf, consultado el 20 de marzo de 2019.

Ministerio de Educación Nacional [MEN] (2013). Lineamientos. Política de Educación Superior inclusiva. Bogotá: autor. https://www.mineducacion.gov.co/1759/articles-357277_recurso.pdf, consultado el 8 de abril de 2019.

Ministerio de Educación Nacional [MEN] (2016). Compendio Estadístico de la Educación Superior colombiana. Bogotá: Imprenta Nacional de Colombia. https://www.mineducacion.gov.co/1759/articles-360739_ recurso.pdf, consultado el 15 de marzo de 2019.

Organización de las Naciones Unidas para la Educación, la Ciencia y la Cultura [Unesco] (2015). Guía para el desarrollo de políticas docentes. París: Unesco. Recuperado de http://unesdoc.unesco.org/ images/0023/002352/235272s.pdf, consultado el 6 de marzo de 2019.

Organización de las Naciones Unidas para la Educación, la Ciencia y la Cultura [Unesco] (2016). La Educación al servicio de los pueblos y el planeta: creación de futuros sostenibles para todos, resumen del informe de seguimiento de la educación en el mundo 2016. París: Unesco. https://unesdoc.unesco.org/ark:/48223/ pf0000245745_spa, consultado el 6 de marzo de 2019.

Organización para la Cooperación y el Desarrollo Económico [OCDE] (2005/2009). Política de educación y formación. Los docentes son importantes: atraer, formar y conservar a los docentes eficientes. París: OCDE Publishing. https://dx.doi.org/10.1787/9789264046276-es, consultado el 13 de marzo de 2019. 
Universidad Pedagógica Nacional [UPN] (2010). Proyecto Educativo Institucional. Bogotá: autor. http:// www.pedagogica.edu.co/userfiles/files/proyecto_educativo_institucional_2010_upn.pdf, consultado el 6 de abril de 2019.

Universidad Pedagógica Nacional [UPN] (2014). Plan de Desarrollo Institucional 2014-2019. Una universidad comprometida con la formación de maestros para una Colombia en paz. Bogotá: Universidad Pedagógica Nacional. http://institucional.pedagogica.edu.co/admin/UserFiles/pdi_upn_2014_2019.pdf, consultado el 4 de abril de 2019.

Universidad Pedagógica Nacional [UPN] (2018). Informe de Gestión 2014-2018. Bogotá: Universidad Pedagógica Nacional.

Universidad del Rosario (2009): "Localidad de Ciudad Bolívar. Universidad, ciencia y desarrollo". Programa de divulgación cientifica, 3 (4). https://www.urosario.edu.co/Universidad-Ciencia-Desarrollo/ur/ Fasciculos-Anteriores/Tomo-IV-2009/Fasciculo-3/ur/Localidad-de-Ciudad-Bolivar/, consultado el 12 de abril de 2019.

\section{Notas biográficas}

Olga Cecilia Díaz Flórez es Doctora en Educación de la Universidad Pedagógica Nacional (UPN) de Colombia, Magíster en Desarrollo Educativo y Social (UPN) y Psicóloga (Universidad Católica de Colombia). Desde el año 2008 es profesora adscrita al Departamento de Posgrado de la Facultad de Educación de la UPN. Fue decana de esta facultad y Subdirectora de Gestión de proyectos - CIUP. Es líder del Grupo de Investigación Educación Superior, Conocimiento y Globalización. Miembro del grupo internacional GIEPES - Grupo Internacional de Estudos e Pesquisa em Educação Superior. Actualmente es la coordinadora académica del equipo de la UPN Colombia en el proyecto TO-INN - From tradition to Innovation in Teacher Training Institutions, financiado por la Unión Europea y liderado por la Universidad de Barcelona. Dentro de sus investigaciones y publicaciones recientes se destacan: el libro Las competencias en la Educación Superior: debates contemporáneos (2018), derivado de su tesis doctoral y el libro colectivo titulado Entre las exigencias de calidady las condiciones de desigualdad: formación inicial de profesores en Colombia (Universidad Pedagógica Nacional y Colciencias, 2018).

Luz Mary Lache Rodríguez es Profesora de la Maestría en Educación y de la Licenciatura en Educación Infantil en la Universidad Pedagógica Nacional de Colombia. Magíster en Educación (UPN) y Licenciada en Tecnología e Informática (UCLA), integrante del grupo de investigación Educación y regionalización en CTeI - GIER, de la misma universidad. Sus intereses investigativos se centran en la formación de maestros, la formación ciudadana y política, la equidad y la diversidad en educación, los ambientes virtuales de aprendizaje, la apropiación social de la ciencia, la tecnología y la innovación, y la incorporación pedagógica de las TIC. Sus últimas publicaciones en coautoría, derivadas de investigación son: el artículo La investigación educativa en contexto en Escuelas Normales Superiores (2019) y el libro La formación de maestros: el oficio del IDEP. Sistematización de la experiencia institucional en formación docente, 1999-2013 (2016). Ha adelantado consultorías para la Fundación Trust for the Americas, la Organización de Estados Iberoamericanos (OEI), la Fundación Internacional de Pedagogía Conceptual Alberto Merani y la Universidad Santo Tomás de Aquino de Bogotá. 
Isabel Garzón Barragán es Doctora en Didáctica de las Ciencias Experimentales de la Universidad de Valencia, España; Magister en Ciencias Físicas y Especialista en Ciencias Físicas de la Universidad Nacional de Colombia y Licenciada en Física de la UPN de Colombia. Profesora adscrita al Departamento de Física de la Facultad de Ciencia y Tecnología de la UPN; Coordinadora del Énfasis de Educación en Ciencias del Doctorado Interinstitucional en Educación. Es integrante del grupo de investigación ALTERNACIENCIAS - Alternativas para la Enseñanza de las Ciencias de la Naturaleza y su área de interés investigativo se encuentra en las relaciones entre la historia, la filosofía y la didáctica de las ciencias en la formación inicial y avanzada de profesores. Entre las publicaciones recientes está el libro colectivo titulado Entre las exigencias de calidady las condiciones de desigualdad: formación inicial de profesores en Colombia (UPN y Colciencias, 2018) y el capítulo de libro El constructivismo en la educación en ciencias como epistemología de la pedagogía, en el libro Epistemología de la pedagogía (UPN y Colciencias, 2018).

Adolfo León Atehortúa Cruz es historiador egresado de las Universidades del Valle (Licenciatura) y Nacional de Colombia (Maestría) y Doctor en Sociología de la Escuela de Altos Estudios en Ciencias Sociales de Paris. Autor de múltiples libros, ensayos y artículos sobre los conflictos socioeconómicos y la historia política de Colombia y América Latina. Entre sus libros más recientes, sobresalen: La Independencia jamás contada (2019), Adiós a las armas (2017), Germán Colmenares. Una nueva historia (2013). Así mismo, entre sus libros más conocidos se destacan: El poder y la sangre, las historias de Trujillo (2005) y Construcción del Ejército Nacional en Colombia (2009). Ha sido Presidente del Consejo Nacional de Planeación, Presidente de la Asociación Colombiana de Universidades (ASCUN) y rector de la Universidad Pedagógica Nacional de Colombia, en donde es actualmente profesor. 